\title{
Efeito do lítio sobre a hiperatividade locomotora induzida pela lesão eletrolítica da região do núcleo mediano da rafe em ratos
}

\author{
Fernanda Augustini Pezzato \\ Diego Bertanha Novais \\ Universidade Estadual Paulista \\ Miriam Garcia-Mijares \\ Universidade de São Paulo \\ Katsumasa Hoshino \\ Universidade Estadual Paulista
}

\begin{abstract}
Resumo
A lesão do núcleo mediano da rafe (NMR) produz sintomas que sugerem validade de face ao episódio maníaco. Esta pesquisa avaliou o efeito do lítio sobre a hiperatividade locomotora induzida por esta lesão. Vinte e um ratos Wistar machos foram submetidos à lesão eletrolítica da região do NMR (LR) e 17 foram submetidos à lesão fictícia (LF). Após recuperação, a atividade locomotora foi avaliada na caixa de atividade (Med Associates/ENV-515). Parte dos animais destes grupos recebeu tratamentos com lítio (47,5 mg/kg/2x dia i.p.) por 10 dias, enquanto o restante foi tratado com salina no mesmo esquema. A reavaliação ao final dos tratamentos demonstrou que o lítio reduziu significantemente a atividade locomotora em relação à avaliação inicial no grupo LR (ANOVA/Bonferroni $p<0,05$ ), tornando-a equivalente aos baixos níveis dos grupos LF. Estes dados sustentam a hipótese de que as manifestações induzidas pela lesão do NMR podem constituir um modelo animal de mania.
\end{abstract}

Palavras chaves: mania; núcleo mediano da rafe; modelos animais; lítio.

\begin{abstract}
Effect of lithium treatment on the locomotor hyperactivity induced by the lesion of the region of the median raphe nucleus in rats. The lesion of the Median Raphe Nucleus (MRN) produces symptoms that suggest face validity for manic episodes. This research evaluated the effect of lithium treatment on the locomotor hyperactivity induced by this lesion. Twenty-one Wistar male rats were submitted to the lesion of the region of the MRN (LR) and 17 were sham lesioned (LF). After recovery, the locomotor activity was evaluated in an activity chamber (Med Associates/ENV-515). A subgroup received lithium $(47.5 \mathrm{mg} / \mathrm{kg} /$ twice a day i.p.) for 10 days, while the other animals received saline in the same schedule. The reevaluation at the end of the treatments showed that only lithium significantly reduced the activity of LR group compared to baseline levels (ANOVA/Bonferroni $p<0.05$ ), making it equivalent to low levels of LF groups. These data support the hypothesis that the behavioral manifestations induced by the lesion of the MRN may constitute an animal model of mania.
\end{abstract}

Keywords: mania; median raphe nucleus; animal models; lithium.

É amplamente reconhecido que os transtornos psicopatológicos ainda necessitam do aumento dos conhecimentos quanto às suas causas, mecanismos e para o desenvolvimento de tratamentos mais eficazes (Gould, Quiroz, Singh, Zarate, \& Manji, 2004; Quiroz, Gould, \& Manji, 2004). Este avanço depende, em parte, da criação e estabelecimento de condições experimentais logicamente fundamentadas que reproduzam em animais de laboratório o fenômeno humano que se quer conhecer (Cryan \& Slattery, 2007; Gould \& Manji, 2004). Esta reprodução, denominada de modelo animal, possibilitou o desenvolvimento de praticamente todos os conhecimentos da medicina experimental a partir de Claude Bernard (1865) e é, hoje, usada praticamente em todas as áreas da pesquisa biológica ligadas à saúde, seguindo regras e preceitos éticos bem definidos, como aponta a revisão de Fagundes e Taha (2004). Seu uso na pesquisa dos problemas psicopatológicos tem sido intenso e objeto de várias revisões (Korff \& Harvey, 2006; Le-Niculescu, Patel, \& Niculescu, 2010; Lipska 2004; Maia \& Frank, 2011; Touma, 2011).

O transtorno bipolar é classificado como um transtorno de humor caracterizado por episódios de depressão maior, mania, hipomania ou misto, separados por períodos de eutimia (humor normal). Um fator limitante para a compreensão da neurobiologia deste transtorno é o número limitado de modelos 
animais disponíveis (Gould \& Einat, 2007), fato compreensível pelas dificuldades de reprodução dos quadros maníacos e da ciclagem entre os episódios depressivos e maníacos. Uma vez que os modelos de depressão maior são disponíveis em maior número, os esforços têm sido dirigidos para o desenvolvimento de modelos animais válidos para o episódio maníaco (Goodwin \& Jamison, 2007). Segundo Lyon (1991), tais modelos de mania devem manifestar, ao menos parcialmente, as seguintes manifestações: 1) hiperatividade; 2) estereotipia; 3 ) aumento da frequência de contatos sexuais; 4) aumento da agressividade; 5) desrespeito à hierarquia social e postura de dominância; 6) prejuízos no julgamento e controle de impulsos; 7) falha na discriminação de estímulos por estímulos irrelevantes; 8) aumento no valor reforçador de estímulos; 9) decréscimo do tempo de sono; 10) irritabilidade; 11) aumento na frequência de contatos sociais gerais; 12) aumento na frequência de vocalizações e 13) alternância de atividades.

A revisão de Einat (2006) revela que atualmente é possível listar três modelos animais para a mania. São eles: a) o da avaliação dos efeitos de estabilizadores de humor sobre a atividade espontânea do animal; b) o dos sintomas maníacos induzidos por psicoestimulantes e c) o dos sintomas maníacos desencadeados pela privação de sono. As induções de sintomas maníacos por psicoestimulantes e pela privação de sono têm sido consideradas válidas por produzirem uma série de sintomas que lhes conferem validade de face para o quadro maníaco (Goodwin \& Jamison, 2007). Os sintomas induzidos por psicoestimulantes envolvem: hiperatividade, estereotipia, maior sensibilidade aos estímulos, alerta, insônia e alterações nos padrões de sono (Einat, 2006; Frey, 2006; O’Donnell \& Gould, 2007). No caso da privação de sono, Gessa, Pani, Fadda e Fratta (1995) descrevem que ratos privados de sono por $72 \mathrm{~h}$ exibem: insônia, hiperatividade, irritabilidade, comportamento agressivo e hipersexualidade. Além disso, tanto a hiperatividade induzida por psicoestimulantes quanto pela privação de sono são sensíveis ao tratamento com lítio (Armani, 2010; Armani et al., 2012; Frey, 2006; Gessa et al., 1995). Entretanto, estes modelos são limitados pela incapacidade de satisfação de todos os critérios de semelhança sintomatológica e pela duração efêmera do quadro maníaco induzido (Malkesman, Austin, Chen, \& Manji, 2009). O modelo da mania induzida por psicoestimulantes, por exemplo, não consegue reproduzir o aumento de apetite da mania e mostra, ao contrário, os conhecidos efeitos anorexígenos destas substâncias (Lyon, 1991). Em relação ao efeito passageiro do quadro induzido, destaca-se que os sintomas deixam de ocorrer em ambos os modelos, passado o efeito da droga ou após curto período de sono dos animais (Gessa et al., 1995). Essa duração efêmera do quadro maníaco impede a investigação dos efeitos de tratamentos experimentais mais longos. O modelo da administração dos estabilizadores de humor sobre a atividade espontânea enfrenta, por sua vez, o problema de ser pouco específico.

A análise geral do problema da falta de modelos mostra que os sintomas maníacos esperados em animais de laboratório envolvem exacerbação comportamental caracterizada pelo aumento da frequência ou intensidade na emissão de diversas atividades, da impulsividade e na dificuldade de concentração.
Tais exacerbações comportamentais sugerem a possibilidade da mania ser um quadro resultante de uma desinibição geral. Nesta perspectiva, o envolvimento do sistema de inibição comportamental do qual participam neurônios serotonérgicos na etiologia deste transtorno merece ser considerado.

O NMR ou superior central (centralis superis) é uma estrutura do tronco cerebral que contém corpos celulares de neurônios cuja neurotransmissão é feita pela 5-hidroxitriptamina (5-HT ou serotonina). Juntamente com o núcleo dorsal da rafe, dá origem à maioria das projeções serotonérgicas ascendentes às áreas límbicas do cérebro que controlam comportamentos emocionais (Beck, Pan, Akawa, \& Kirby, 2004). Estes núcleos, juntamente com os demais componentes do sistema serotonérgico central foram estudados na busca da compreensão dos sistemas de inibição e desinibição comportamental (Andrade, 1997; Graeff \& Silveira Filho, 1978; Gray, 1982; 1987). Pesquisas posteriores investigaram o envolvimento do NMR nos mecanismos geradores do medo e da ansiedade (Andrade, Silva, Silva, \& Graeff, 1999; Andrade \& Graeff, 2001; Dominguez, Cruz-Morales, Carvalho, Xavier, \& Brandão, 2003). Atribui-se também importante papel ao NMR na regulação do sono, uma vez que este estaria relacionado à modulação exercida pela luz sobre o ritmo circadiano por meio de projeções ao núcleo supraquiasmático (Meyer-Bernstein \& Morin, 1996; MeyerBernstein \& Morin, 1999; Muscat, Tischler, \& Morin, 2005).

Pesquisas realizadas em animais de laboratório com lesão do NMR descrevem a ocorrência de um conjunto de manifestações semelhantes às apontadas como maníacas por Lyon (1991), quais sejam: a) hiperatividade (De Paula, Uga, \& Hoshino, 2000; Jacobs, Wise, \& Taylor, 1974; Uga, De Paula, \& Hoshino, 2000); b) estereotipia (De Paula et al., 2000; Hoshino, Uga, \& De Paula., 2004); c) facilitação no comportamento sexual (Albinsson, Andersson, Andersson, Vega-Matuszczyk, \& Larsson, 1996); d) agressividade e desrespeito à hierarquia social (File, Hyde, \& Macleod; 1979); e) impulsividade (Fletcher, 1995; Le, Harding, Juzytsch, Fletcher, \& Shaham, 2002); f) alterações na aprendizagem e controle de estímulos (Asin, Wirtshafter, \& Kent, 1979; 1980; Asin, Wirtshafter, \& Fibiger, 1985; Lima, 2002); g) aumento da frequência de comportamentos hedônicos (Pezzato \& Hoshino, 2004; Wogar, Bradshaw, \& Szabadi; 1991) e h) diminuição do tempo total de sono (Arpa \& Andrés, 1993).

Apesar dos vários estudos existentes a respeito dos efeitos comportamentais da lesão da região do NMR, não existem publicações estabelecendo especificamente que eles são manifestações maníacas. Para isto, e subsequente uso como modelo animal, tais efeitos precisam da demonstração de atendimento aos demais critérios formais de validação estabelecidos por Willner (1991). Uma vez que existem similaridades sintomatológicas das manifestações dos animais lesados com os do quadro maníaco humano - indicando validade de face - é justificável a proposição de averiguar o atendimento ao critério de validade preditiva. Este critério que estabelece a necessidade do modelo ter a capacidade de responder de maneira semelhante a tratamentos de mesma natureza (inclusive a serem feitas no futuro, fato definível como predição). A avaliação desse critério é feita com base nos efeitos da administração de fármacos usados classicamente na terapêutica. Nesse aspecto, o carbonato 
de lítio tem sido eleito para avaliar a validade preditiva dos modelos de mania (O’Donnell \& Gould, 2007), pois ele é capaz de reduzir sintomas maníacos, bem como prevenir ciclagens entre mania e depressão (Lyon, 1991; Muller-Oerlinghausen, Berghofer, \& Bauer, 2002).

A relevância da criação de modelos animais para a compreensão de processos psicopatológicos levou o presente estudo a avaliar o efeito da administração de lítio na hiperatividade locomotora induzida pela lesão da região do NMR, com o intuito de subsidiar a validade da hipótese de que o quadro comportamental induzido pela lesão do NMR pode vir a constituir um modelo animal válido de mania.

\section{Método}

O presente estudo foi aprovado pelo Comitê de Ética na Pesquisa da Instituição (processo $\mathrm{n}^{\mathrm{o}}$ 6171/46/01/11) e sua execução seguiu a lei 11794/08 de Procedimentos para uso científico de animais (Brasil).

\section{Animais}

Foram utilizados 38 ratos Wistar machos, provenientes do Biotério Central da Universidade Estadual Paulista. Eles foram fornecidos com 45 dias de vida e alojados em grupos de quatro animais em caixas de poliuretano semitransparente, com assoalho coberto de maravalha, mantidos em ambiente com ciclos claro/ escuro de 12h (luz das 7:00 às 19:00 h) e temperatura de 25-26 $6^{\underline{0}}$ $\mathrm{C}$, tendo água e ração padronizada ad libitum. Ao atingirem 90 dias e peso médio de $350 \mathrm{~g}$, os animais foram submetidos à cirurgia para início dos procedimentos experimentais (Hoshino et al., 2004).

\section{Equipamentos}

Lesão eletrolítica do NMR: lesionador eletrônico (3500 Lesion Producing Device), eletrodo de eletrocoagulação de fio de níquel-cromo (0,12 $\mathrm{mm})$, aparelho estereotáxico para pequenos animais (David-Kopff - modelo 900).

Avaliação da atividade locomotora: duas Caixas de Atividade de Campo Aberto (ENV-515) em policarbonato acrílico transparente, de medidas 42,5 $\mathrm{cm}$ x 42,5 cm x 30,5 cm (Med- Associates). Feixes de raios infravermelhos, dispostos em diferentes alturas deste aparato possibilita a detecção e contagem automática da locomoção do animal (sensibilidade de um centímetro), o tempo de imobilidade, o comportamento de levantar-se nas patas posteriores. O controle das sessões experimentais e o registro dos dados foram feitos em um computador IBM conectado às interfaces das caixas.

\section{Fármacos}

Cloridrado de ketamina (Dopalen $\AA /$ Vetbrands), xilazina (Anasedan $\AA /$ Vetbrands), Xilocaína ${ }^{\circledR}$ com vasoconstritor (ASTRA Química e Farmacêutica Ltda), pentabiótico veterinário (Fort Dodge), diclofenaco sódico (Cataflan®/NOVARTIS) e Carbonato de Lítio (Carbolitium $\AA /$ Eurofarma).

\section{Procedimentos}

Lesão eletrolítica da região do NMR (Hoshino et al., 2004): os animais foram divididos em dois grupos, sendo um deles submetidos à lesão eletrolítica do NMR (grupo LR, $n=21$ ) e o restante dos animais submetido à lesão fictícia, constituindo o grupo controle (grupo LF, $n=17$ ). Os animais foram anestesiados com ketamina (i.p. $75 \mathrm{mg} / \mathrm{kg}$ ) e sedados com xilazina (i.m. $1 \mathrm{mg} / \mathrm{kg}$ ). Após a perda do reflexo óculopalpebral, anestésico local (xilocaína $2 \%$, com vasoconstrictor) foi injetado subcutaneamente na região da cirurgia. Os animais foram fixados ao aparelho estereotáxico e a calota craniana exposta. O eletrodo isolado eletricamente em sua extensão, exceto o milímetro final, foi inserido com um ângulo de 30 graus no plano ântero-posterior através de uma trepanação no osso interparietal, usando-se as seguintes coordenadas do atlas estereotáxico de Paxinos e Watson (1986): A: 1,0 e 1,8; LR: 0,0 e $\mathrm{H}=1,6 \mathrm{~mm}$ tomando-se a referência auricular. Corrente DC de $4 \mathrm{~mA}$ por 15 segundos foi aplicada para a eletrocoagulação nos animais experimentais (grupo LR), enquanto nos animais controles (grupo LF), o eletrodo foi inserido próximo ao núcleo $(\mathrm{H}=2,8)$ e retirado sem aplicação da corrente eletrocoagulante. Os animais foram suturados, a assepsia cuidada, tratados com pentabiótico veterinário (dose única i.m. $0,2 \mathrm{ml} / \mathrm{kg}$ ) e mantidos individualmente em gaiolas-viveiro. Nos três primeiros dias de recuperação pós-operatória foi administrado analgésico em gotas (diclofenaco sódico a $1 \mathrm{ml} / \mathrm{kg}$ animal). Após período de recuperação pós-cirúrgica de sete dias os testes e tratamentos foram iniciados.

\section{Avaliação da atividade locomotora e tratamentos}

1) Teste 1- linha de base (LB): cinco minutos antes de cada sessão os animais foram levados em suas gaiolas-viveiro à sala de experimentação. Os animais foram transferidos gentilmente para a caixa de medida da atividade e a locomoção espontânea computada por 15 minutos contínuos, sendo a ordem dos animais testados estabelecida aleatoriamente por sorteio. As avaliações foram realizadas no período da tarde (entre 12:00 e 15:00h), permanecendo a luz da sala acesa durante todo o período. $\mathrm{O}$ equipamento foi limpo com álcool $70 \%$ após o teste de cada animal, impedindo a permanência de odores.

2) Tratamento com carbonato de lítio ou solução salina: 10 animais do grupo LR e 9 animais do grupo LF foram tratados por 10 dias por carbonato de lítio $(47.5 \mathrm{mg} / \mathrm{Kg} / \mathrm{dia}$, referente a $1,29 \mathrm{mEq} / \mathrm{kg})$ diluído em solução salina $(0,9 \%)$ acidificada para neutralização do $\mathrm{pH}$ (ajustado para 7,4 pela adição de $\mathrm{HCl} 2 \mathrm{~N}$ ) (Armani, 2010; Armani et al., 2012), dividido em duas doses diárias administradas intraperitonealmente as $9 \mathrm{~h}$ e as $21 \mathrm{~h}$. Esta dose foi estabelecida como segura visto que avaliações piloto com doses maiores mostraram induzir quadros de intoxicação e óbito. O restante dos animais foi tratado com administração intraperitoneal de volumes correspondentes de solução salina nos mesmos horários ( $n=11$ do grupo LR e $n=8$ do grupo LF).

3) Teste 2 - Avaliação final: no décimo dia de tratamento a atividade locomotora foi reavaliada, seguindo o mesmo procedimento do Teste 1(LB). Neste dia os animais receberam os tratamentos e foram testados após intervalo padronizado de tempo (Armani, 2010; Armani, et al., 2012) de duas horas após a última injeção.

\section{Análise dos dados}


A atividade locomotora dos grupos foi avaliada por uma Anova factorial mista de duas vias com um fator dependente ou de medida repetida com dois níveis (LB e avaliação final) e um fator independente ou de grupo com quatro níveis (grupos LR-lítio, LR-salina, LF-lítio, LF-salina).

Os efeitos estatisticamente significativos foram analisados pelo teste post hoc de Bonferroni (pelo método de combinação de somas residuais de quadrados entre-intra). Foi adotado o nível de significância de $p<0,05$. Todas as análises foram realizadas pelo software STATISTICA ${ }^{\circledR}$ v.10 (StatSoft).

\section{Resultados}

A Figura 1 mostra as médias da atividade locomotora dos grupos LR e LF tratados com carbonato de lítio e solução salina, expressos em termos de distância percorrida em centímetros, tanto nas condições LB como na avaliação final após a administração do carbonato de lítio. A análise de variância demonstrou diferenças significativas entre as atividades locomotoras dos grupos (efeito principal para o fator independente; $F(3,34)=$ 19,56; $p<0,001$ ), entre as medidas obtidas neste parâmetro na LB e na avaliação final (efeito principal para o fator dependente;
$F(1,34)=20,29 ; p<0,001)$ e interação entre o fator independente e o dependente, isto é, entre os grupos e a medida repetida $(F(3,34)=5,11 ; p=0,005)$.

Como indicado na Figura 1, a análise post hoc de Bonferroni mostrou que as médias da atividade locomotora na LB foram significativamente maiores nos grupos com lesão do NMR do que nos grupos com LF ( $p<0,001$ para as comparações LR-lítio vs LF-lítio, LR-lítio vs LF-sal, LR-sal vs LF-lítio e LR-sal vs LF-sal). Não foram observadas outras diferenças entre grupos nessa fase.

Os animais do grupo LR-lítio mostraram diminuição da atividade locomotora após o tratamento com lítio $(p<0,001$ para a comparação entre LB e avaliação final). Para o resto dos grupos não foram observadas alterações significativas entre as medidas obtidas de LB e as obtidas na avaliação final. As diferenças entre os grupos nessa fase confirmam essas observações: houve diferenças significativas na atividade locomotora exibida pelos animais do grupo LR-sal e os grupos com lesão fictícia $(p<$ 0,001 para ambas as comparações), mas não entre LR-lítio e os grupos com lesão fictícia. Os demais contrastes não apresentaram significância estatística.

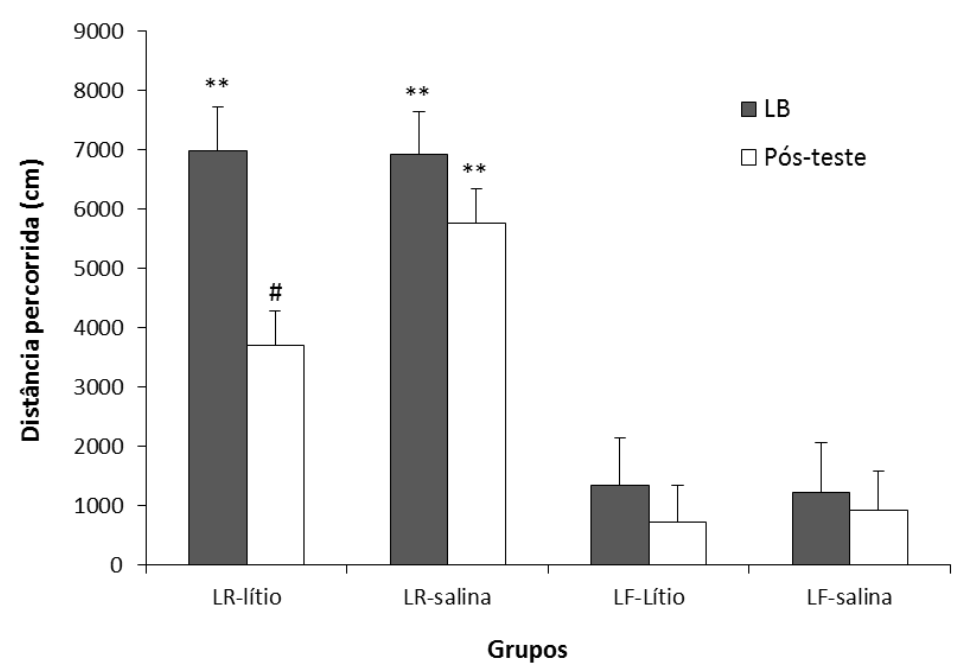

Figura 1. Efeito do lítio sobre a atividade locomotora de ratos submetidos a lesão do NMR. Os valores representam as médias (+EPM) da atividade locomotora (distancia percorrida $-\mathrm{cm}$ ) dos grupos tratados com lítio (LR-lítio e LF-lítio) ou com salina (LR- salina LF-salina) nas sessões de linha de base (LB) e décimo dia de tratamento (pós-teste). Os animais dos grupos LR-lítio e LR-salina foram submetidos a lesão do NMR e os animais dos grupos LF-lítio e LF-salina sofreram lesão fictícia antes das medidas de LB serem coletadas. **p $<0,001$ para diferenças da atividade dos grupos LR-lítio e LR-salina em relação aos grupos LF-lítio e LF- salina. ${ }_{p} p<0.001$ para diferenças entre LB e pós-teste.

\section{Discussão}

O resultado principal obtido no presente estudo mostra que o aumento da atividade locomotora induzida pela lesão do NMR é reduzido de maneira estatisticamente significante após um período de tratamento com o lítio. Os dados indicam também que essa redução equipara a atividade locomotora dos animais lesados aos dos animais com lesão fictícia. Fato similar não foi observado nos animais lesados tratados com salina.

O aumento significativo da atividade locomotora constatada nos animais submetidos à lesão do NMR era esperado, uma vez que este efeito é amplamente conhecido e frequentemente reproduzido (De Paula et al., 2000; Jacobs et al., 1974; Uga et al., 2000). Uma vez que as médias da atividade locomotora nos ratos lesados praticamente quintuplicaram em relação à atividade dos animais controles com LF, pode-se considerar com segurança 
que os animais lesados manifestaram hiperatividade locomotora. Adicionalmente, a constatação de que a hiperatividade locomotora manifesta na linha de base pelos animais com LR se reduziu significativamente após o tratamento com lítio, a ponto das diferenças em relação aos grupos com LF perderem a significância estatística, permite aceitar que a lesão induz hiperatividade de mesma natureza que as induzidas em outros modelos experimentais aceitos da mania (Einat, 2006; Gessa et al., 1995; Goodwin \& Jamison, 2007) que, por sua vez, se assemelha à hiperatividade dos pacientes maníacos. Por extensão, pode-se afirmar que os dados obtidos dão suporte à validade preditiva e fundamenta a proposição de que o quadro comportamental induzido pela lesão do NMR constitui um modelo experimental de mania. Esta interpretação encontra suporte nos outros efeitos desencadeados pela lesão, tais como o aumento na emissão de estereotipias, na impulsividade, diminuição do tempo de sono, facilitação sexual, aumento na frequência de comportamentos hedônicos, dentre outros (Albinsson et al., 1996; Arpa \& Andrés, 1993; Fletcher, 1995; Hoshino et al., 2004; Le et al., 2002; Pezzato \& Hoshino, 2004; Wogar et al., 1991).

Os resultados obtidos mostram também que os animais LR tratados com lítio reduziram a atividade locomotora, porém, sem diferença estatisticamente significativa quando comparada ao dos animais lesados tratados com salina. Esta falta de significância, quando considerada isoladamente, indica ineficácia do tratamento com lítio. Esse dado, por ser fundamental para a conclusão do presente trabalho, precisa ser analisado com cuidado uma vez que é contraditório aos mostrados pela literatura. Primeiro, é preciso considerar que o tratamento com carbonato de lítio não tem eficiência completa e universal, mostrando-se ineficaz, enquanto monoterapia na redução de sintomas maníacos, em cerca de $25 \%$ a $30 \%$ dos pacientes (Stokes, Shamoian, Stoll, \& Patton, 1971; WGBD, 2004). Tal limitação terapêutica é, justamente, o propósito para a procura de modelos animais que possam auxiliar na busca de novos tratamentos mais eficazes. Segundo, deve-se considerar que a própria literatura registra a obtenção de êxito relativamente baixo ou mesmo contraditório quanto à eficácia do lítio em reduzir a hiperatividade induzida em ratos com o uso de psicoestimulantes, conforme evidenciado na revisão de O'Donnell e Gould (2007). Terceiro, a falta de significância estatística na diferença em questão mostra decorrer, ao menos parcialmente, do abaixamento relativo, porém não significante, da média do grupo LR tratado com salina, como se pode constatar na comparação com o valor da sua linha de base. Tal fato pode ser atribuído à adaptação do organismo à lesão, efeito descrito por Jacobs et al. (1974) ou à habituação à novidade da situação na reexposição dos animais ao teste, fenômeno que é bastante conhecido. Embora tais fatores também tenham afetado os animais do grupo LR tratados com lítio, uma redução adicional devida à ação do fármaco é inegável, pois: a) a atividade locomotora do grupo LR-lítio, mas não do grupo LR-salina, foi significantemente menor na avaliação final do que na LB e b) como já mencionado, a atividade locomotora do grupo LR-lítio não foi estatisticamente diferente daqueles mostrados pelos grupos com LF na avaliação final. Tal fato não foi observado no grupo LR-salina.
Duas categorias de problemas referentes ao tratamento por lítio descritas na literatura se encaixam no rol das interpretações aventadas para explicar a ausência de significância nas diferenças da atividade locomotora dos ratos lesados após tratamentos com lítio e salina: a) a variabilidade do efeito do lítio intersujeitos, visível na magnitude dos desvios-padrão; b) a dose de lítio administrada no experimento. No que se refere à variabilidade intersujeitos, deve-se considerar que a metabolização do lítio é diversificada, podendo a mesma dosagem resultar em diferentes valores séricos, denominados litemias. Tal diversidade de efeitos entre sujeitos, espécies e linhagens de roedores, juntamente com a instabilidade de doses utilizadas - que variam a cada pesquisa - também são apontadas na revisão realizada por O’Donnell e Gould (2007) como responsáveis pelas inconsistências nos resultados de tratamento crônico com lítio em animais de laboratório. Estes mesmos autores sugerem que doses séricas terapêuticas de lítio em ratos para administração i.p. ou subcutânea diferem da dosagem humana, sendo obtidas com doses superiores, entre 1,5 e $3 \mathrm{mEq} / \mathrm{kg}$.

Considerando a estreita janela terapêutica do lítio - que em humanos varia entre 0,5 e 1,2 mEq/kg - doses padronizadas por peso podem resultar tanto em efeito nulo, quanto terapêutico ou tóxico em diferentes animais de uma mesma linhagem (O’Donnell \& Gould, 2007). A dose de lítio utilizada no tratamento dos animais da presente pesquisa foi de $1,29 \mathrm{mEq} /$ $\mathrm{kg}$ por dia, podendo ser considerada como subterapêutica em ratos, o que aumentaria a probabilidade da ineficácia ou eficácia reduzida em alguns animais. Entretanto, em estudos pilotos de nosso laboratório doses ligeiramente mais altas mostraramse tóxicas e letais aos animais. O risco de intoxicação é um importante fator a ser considerado e podendo se tornar crítico quando o parâmetro avaliado é a atividade locomotora, uma vez que resulta em sonolência e letargia (Kaplan, Sadock, \& Grebb, 1997) - podendo acusar valores falso-positivos em relação à eficácia da droga.

O pouco conhecimento acerca dos mecanismos de ação do lítio reduz as possibilidades de previsão dos efeitos da administração crônica em animais submetidos à inativação do NMR. Sabe-se que o lítio exerce diversas ações bioquímicas relacionadas à liberação cálcio-dependente de noradrenalina e dopamina, ao bloqueio de síntese de fosfatidilinositol e modificação de respostas mediadas pelo sistema adenilatociclase e AMPc (Gorenstein \& Scavone, 1999). Adicionalmente, propriedades antidepressivas do lítio somadas a dados de pesquisas (Bourin, Hascoet, Colombel, Redrobe, \& Baker, 1996; Scheuch et al., 2010; Subhash, Vinod, \& Srinivas, 1999), fornecem indícios do envolvimento do sistema serotonérgico central, do qual o NMR faz parte, na eficácia desta droga. Scheuch et al., (2010), por exemplo, investigaram os efeitos do cloreto de lítio ( $\mathrm{LiCl}, 1 \mathrm{mM})$ na neurotransmissão serotoninérgica proveniente dos núcleos da rafe em ratos. Os tratamentos agudos e os prolongados ( $8 \mathrm{~h}$ e 14 dias) resultaram respectivamente em $20 \%$ e $23 \%$ de aumento na liberação de 5-HT, mas não influenciaram na absorção ou na recaptação deste neurotransmissor. Os resultados demonstraram também que o lítio pode modificar a expressão gênica de TPH2- enzima limitante de biossíntese de 5HT- resultando em $45 \%$ de redução 
de THP2mRNA e 31\% de redução dos níveis protéicos de TPH2. Logo, se o efeito do lítio está ao menos parcialmente relacionado ao aumento de disponibilidade de 5-HT nos núcleos da rafe, é possível admitir que parte de sua ação fica excluída nos animais lesionados no NMR. Em tal caso, a ação do lítio que se expressa com a intermediação da neurotransmissão dopaminérgica e/ ou noradrenérgica (Gorenstein \& Scavone, 1999) pode ser a responsável pela redução observada no presente estudo.

\section{Conclusões}

O estabelecimento de que a hiperatividade locomotora induzida pela lesão do NMR mostra ser reduzida pelo tratamento com lítio permite deduzir que efeito semelhante deverá ser observado caso alguma substância com propriedade antimaníaca já conhecida ou desconhecida for administrada. Este dado dá fundamentação à validade preditiva e consolida a hipótese dos animais com este tipo de lesão ser modelo animal do transtorno maníaco. O fato da lesão do NMR reproduzir, em um mesmo animal, vários sintomas similares aos que se verificam na mania, dá suporte a esta conclusão.

Por fim, cabe apontar um fato que a proposição do modelo de mania baseado nos animais com lesão do NMR traz. Como dito anteriormente, a atividade serotonérgica do NMR é considerada parte de um sistema geral de inibição. Diferentemente, os modelos de mania existentes, baseados na administração de psicoestimulantes - principalmente anfetaminas - envolvem neurotransmissão dopaminérgica e noradrenérgica (Gorenstein \& Scavone, 1999). A constatação de que uma manifestação maníaca pode decorrer tanto da ativação dos mecanismos centrais de alerta, como da inativação ou rebaixamento da atividade nos mecanismos inibidores, permite a conceituação de que o equilíbrio entre estes mecanismos é a condição normal de vida e que seu desequilíbrio pode resultar na excitação maníaca ou em quadros depressivos. Estas deduções demonstram o alto valor heurístico dos modelos animais que os psicólogos também ajudam a criar e estudar.

\section{Referências}

Albinsson, A., Andersson, G., Andersson, K., Vega-Matuszczyk, J., \& Larsson K. (1996). The effects of lesions in the mesencephalic raphe systems on male rat sexual behavior and locomotor activity. Behavioural Brain Research, $80(1-2), 57-63$.

Andrade, T. G. (1997). Participação do núcleo mediano da rafe na modulação de respostas comportamentais, viscerais, endócrinas e imunológicas, frente a estímulos aversivos (Tese de Doutorado não publicada). Universidade de São Paulo, Ribeirão Preto.

Andrade, T. G., Silva, A. M., Silva C. L., \& Graeff, F. G. (1999). Effect of electrolytic lesion of the median raphe nucleus on behavioral and psychological measures of stress. Acta Phisiologica, Pharmacologica et Therapeutica Lationoamericana, 49, 279-289.

Andrade, T. G., \& Graeff, F. G. (2001). Effect of electrolytic and neurotoxic lesions of the median raphe nucleus on anxiety and stress. Pharmacology, Biochemistry and Behavior, 70, 1-14.

Armani, F. (2010). Reversão de comportamentos relacionados à mania induzidos pela privação de sono paradoxal pelo lítio e tamoxifeno em camundongos (Dissertação de Mestrado não publicada). Universidade Federal de São Paulo, São Paulo.
Armani, F., Andersen, M. L.; Andreatini, R., Frussa-Filho, R., Tufik, S., \& Galduroz, J. C. F. (2012). Successful combined therapy with tamoxifen and lithium in a paradoxical sleep deprivation-induced mania model. CNS Neuroscience \& Therapeutics, 18(2), 119-125. doi: 10.1111/j.17555949.2010.00224.x

Arpa, J., \& Andrés, I. (1993). Re-examination of the effects of raphe lesions on the sleep/wakefulness cycle states in cats. Journal of Sleep Research, 2(2), 96-102.

Asin, K. E., Wirtshafter, D., \& Kent, E. W. (1979). Impaired patterned responding in rats with electrolytic median raphe lesions. Physiology \& Behavior, 23(4), 803-806.

Asin, K. E., Wirtshafter, D., \& Kent, E. W. (1980). The effects of electrolytic median raphe lesions on two measures of latent inhibition. Behavioral and Neural Biology, 28(4), 408-417.

Asin, K. E., Wirtshafter, D., \& Fibiger, H. C. (1985). Eletrolytic, but not 5,7dihydroxytryptamine, lesion of the nucleus medianus raphe impair acquisition of a radial maze task. Behavioral and Neural Biology, 44(3), 415-424.

Beck, S. G., Pan, Y. Z., Akawa, A. C., \& Kirby, L. G. (2004). Median and dorsal raphe neurons are not eletrophysiologically identical. Journal of Neurophysiology, 91(2), 994- 1005.

Bourin, M., Hascoet, M., Colombel, M. C., Redrobe, J. P., \& Baker, G. B. (1996). Differential effects of clonidine, lithium and quinine in the forced swimming test in mice for antidepressants: possible roles of serotoninergic systems. European Neuropsychopharmacology, 6(3), 231-236.

Cryan, J. F., \& Slattery, D. A. (2007). Animal models of mood disorders: recent developments. Current Opinion in Psychiatry, 20, 1-7.

De Paula, H. M. G., Uga, D. A., \& Hoshino, K. (2000). Hiperatividade locomotora em ratos com lesão do núcleo mediano da rafe: um modelo experimental de compulsão? In FESBE (Org.), Resumos da XV Reunião Anual da FESBE (p.182). Caxambu: Autor.

Dominguez, R., Cruz-Morales, S. E., Carvalho, M. C., Xavier, M., \& Brandão, M. L. (2003). Sex differences in serotonergic activity in dorsal and median raphe nucleous. Physiology \& Behavior, 80(2-3), 203-210.

Einat, H. (2006). Modeling facets of mania - new directions related to the notion of endophenotypes. Journal of Psychopharmacology, 20(5), 714-722.

Fagundes, D. J., \& Taha, M. O. (2004). Modelo animal de doença: critérios de escolha e espécies de animais de uso corrente. Acta Cirúrgica Brasileira, 19 (1), 59-65.

File, S. E.; Hyde, J. R. G., \& Macleod, N. K. (1979). 5,7-Dihydroxytryptamine lesions of dorsal and median raphé nuclei and performance in the social interaction test of anxiety and in a home-cage aggression test. Journal of Affective Disorders, 1(2), 115-122.

Fletcher, P. J. (1995). Effects of combined or separate 5,7-dihydroxytryptamine lesions of the dorsal and median raphe nuclei on responding maintained by a DRL 20s schedule of food reinforcement. Brain Research, 675, 45-54.

Frey, B. N., Valvassori, S. S., Reus, G. Z., Martins, M. R., Petronilho, F. C., Bardini, K., ... \& Quevedo, J. (2006). Effects of lithium and valproate on amphetamine-induced oxidative stress generation in an animal model of mania. Journal of Psychiatry \& Neuroscience, 31, 326-332.

Gessa, G. L., Pani, L., Fadda, P., \& Fratta, W. (1995). Sleep deprivation in the rat: an animal model of mania. European Neuropsychopharmacology, 5(Suppl), 89-93.

Goodwin, F. K., \& Jamison, K. R. (2007). Manic-depressive illness: bipolar disorders and recurrent depression ( $2^{\mathrm{a}}$ ed.). Nova Iorque: Oxford University Press.

Gorenstein, C., \& Scavone, C. (1999). Avanços em psicofarmacologia mecanismos de ação de psicofármacos hoje. Revista Brasileira de Psiquiatria, 21(1), 64-73.

Gould, T. D., Quiroz J. A., Singh, J., Zarate, C. A., \& Manji, H. K. (2004). Emerging experimental therapeutics for bipolar disorder: insights from the molecular and cellular actions of current mood stabilizers. Molecular Psychiatry, 9, 734-755 
Gould, T. D., \& Manji, H. K. (2004). The molecular medicine revolution and psychiatry: bridging the gap between basic neuroscience research and clinical psychiatry. Journal of Clinical Psychiatry, 65(5), 598-604.

Gould, T. D., \& Einat, H. (2007). Animal models for bipolar disorder and mood stabilizer efficacy: a critical need for improvement. Neuroscience and Biobehavioral Reviews; Editorial, 31(6), 825-831.

Graeff, F. G., \& Silveira Filho, G. (1978). Behavioral inhibition induced by electrical stimulation of the median raphe nucleous of the rat. Psysiology \& Behavior, 71, 477-484.

Gray, J.A. (1982). The neuropsycology of anxiety. Nova Iorque: Oxford University Press.

Gray, J. A. (1987). The psychology of fear and stress. Cambridge, Cambridge University Press.

Hoshino, K., Uga, D. A., \& De Paula, H. M. G. (2004). The compulsive-like aspect of the head dipping emission in rats with chronic electrolytic lesion in the area of the median raphe nucleus. Brazilian Journal of Medical and Biological Research, 37, 245-250.

Jacobs, B. L., Wise, W. D., \&. Taylor, K. M. (1974). Differential behavioral and neurochemical effects following lesions of the dorsal or median raphe nuclei in rats. Brain Research, 79(3)25, 353-361.

Kaplan, H. I.; Sadock, B. J., \& Grebb, J. A. (1997). Compêndio de Psiquiatria: Ciências do comportamento e psiquiatria clínica ( $7^{\mathrm{a}}$ ed., D. Batista, Trad.). Porto Alegre: Artmed.

Korff, S., \& Harvey, B. H. (2006). Animal models of obsessive-compulsive disorder: rationale to understanding psychobiology and pharmacology. The Psychiatric Clinics of North America, 29(2), 371-90.

Le, A. D., Harding, S., Juzytsch, W., Fletcher, P. J., \& Shaham, Y.(2002). The role of corticotropin-releasing factor in the median raphe nucleus in relapse to alcohol. The Journal of Neuroscience, 22(18), 7844-7849.

Le-Niculescu, H., Patel, S. D., \& Niculescu, A. B. (2010). Convergent integration of animal model and human studies of bipolar disorder (manic-depressive illness). Current Opinion in Pharmacololy, 10(5), 594-600.

Lima, V. A. (2002). O núcleo mediano da rafe no medo condicionado: aspectos comportamentais, autonômicos e motores. (Tese de Doutorado). Universidade de São Paulo, Ribeirão Preto.

Lipska, B. K. (2004). Using animal models to test a neurodevelopmental hypothesis of schizophrenia. Journal of Psychiatry \& Neuroscience, 29(4), 282-286.

Lyon, M. (1991). Animal models of mania and schizophrenia. In P. Willner (Org.), Behavioral Models in Phychopharmachology: Theoretical, industrial and clinical perspectives (pp.253-310). Cambridge: Cambridge University Press.

Maia, T. V., \& Frank, M. J. (2011). From reinforcement learning models to psychiatric and neurological disorders. Nature Neuroscience, 14(2), 154-62.

Malkesman, O., Austin, D. R., Chen, G., \& Manji, H. K. (2009). Reverse translational strategies for developing animal models of bipolar disorder. Disease Models \& Mechanisms, 2(5-6), 238-245.

Meyer-Bernstein, E. L., \& Morin, L. P. (1996). Differential serotonergic innervation of the suprachiasmatic nucleus and the intergeniculate leaflet and its role in circadian rhythm modulation. The Journal of Neuroscience, 16(6), 2097-2111.

Meyer-Bernstein, E. L., \& Morin, L. P. (1999). Electrical stimulation of the median or dorsal raphe nuclei reduces light-induced FOS protein in the suprachiasmatic nucleous and causes circadian activity rhythm phase shifts. Neuroscience, 92(1), 267-79.

Muller-Oerlinghausen, B., Berghofer, A., \& Bauer, M. (2002): Bipolar disorder. The Lancet, 359, 241-247.

Muscat, L., Tischler, R. C., \& Morin, L. P. (2005). Functional analysis of the role of the median raphe as a regulator of hamster circadian system sensitivity to light. Brain Research, 17, 1044(1), 59-66.

O'Donnell, K. C., \& Gould, T. D. (2007). The behavioral actions of lithium in rodent models: leads to develop novel therapeutics. Neuroscience and Biobehavioral Reviews, 31, 932-962.

Paxinos, G., \& Watson, C. (1986). The rat brain in stereotaxic coordinates $\left(2^{\mathrm{a}}\right.$ ed.). Londres: Academic Press.

Pezzato, F. A., \& Hoshino, K. (2004). Número de respostas e extinção em ratos com lesão do núcleo mediano da rafe. In ABPMC (Org.), XIII Encontro da Associação Brasileira de Psicoterapia e Medicina Comportamental e II Congresso Internacional da Association for Behavior Analisis (p.219-220). Campinas: Autor.

Quiroz, J. A., Gould, T. D., \& Manji, H. K. (2004). Molecular effects of lithium. Molecular Interventions, 4(5), 259-272.

Scheuch, K., Höltje, M., Budde, H., Lautenschlager, M., Heinz, A., Ahnert-Hilger, G., \& Priller, J. (2010). Lithium modulates tryptophan hydroxylase 2 gene expression and serotonin release in primary cultures of serotonergic raphe neurons. Brain Research, 1307(11), 14-21.

Stokes, P. E., Shamoian, C. A., Stoll, P. M., \& Patton, M. J. (1971). Efficacy of lithium as acute treatment of manic-depressive illness. The Lancet, 297(7713), $1319-1325$.

Subhash, M. N., Vinod, K. Y., \& Srinivas, B. N. (1999). Differential effect of lithium on 5-HT1 receptor-linked system in regions of rat brain. Neurochemistry International, 35(4), 337-343. doi :10.1016/S01970186(99)00079-0.

Touma, C. (2011). Stress and affective disorders: animal models elucidating the molecular basis of neuroendocrine-behavior interactions. Pharmacopsychiatry, 44(Suppl 1:S), 15-26.

Uga, D. A., De Paula, H. M. G., \& Hoshino, K. (2000). Hiperatividade locomotora da lesão do n. mediano da rafe e susceptibilidade ao pânico da crise audiogênica em ratos. In FESBE (Org.), Resumos da XV Reunião Anual da FESBE (p.120). Caxambu: Autor.

Willner, P. (1991). Behavioral models in psychopharmacology. In P. Willner (Org.), Behavioral Models in Phychopharmachology: theoretical, industrial and clinical perspectives (pp. 3-18). Cambridge: Cambridge University Press.

Wogar, M. A., Bradshaw, C. M., \& Szabadi, E. (1991). Evidence for an involvement of 5-hydroxytryptaminergic neurones in the maintenance of operant behaviour by positive reinforcement. Journal Psychopharmacology, 105(1), 305-315. 
128 F. A. Pezzato, D. B. Novais, M. Garcia-Mijares \& K. Hoshino

Fernanda Augustini Pezzato, doutoranda em Psicologia Experimental na Universidade de São Paulo, é professora Substituta na Universidade Estadual Paulista. Telefone: (11) 38184444 Ramal: 208. E-mail: fernandapezzato@usp.br

Diego Bertanha Novais, graduando em Psicologia na Universidade Estadual Paulista. E-mail: bertanhanovais@ hotmail.com

Miriam Garcia-Mijares, doutora em Neurociências e Comportamento pela Universidade de São Paulo, é professora na Universidade de São Paulo. E-mail: mgarciam@usp.br

Katsumasa Hoshino, é professor Titular do Departamento de Ciências Biológicas, Universidade Estadual Paulista. E-mail: kt.hoshino@gmail.com

Recebido em 14.jul.11 Revisado em 21 .abr. 